Special Issue of the 6th International Congress \& Exhibition (APMAS2016), Maslak, Istanbul, Turkey, June 1-3, 2016

\title{
Magnetic Properties of $\mathrm{La}_{3} \mathrm{Ga}_{5.5} \mathrm{Ta}_{0.5} \mathrm{O}_{14}$ Single Crystals Doped with $\mathrm{Sm}^{3+}$ and $\mathrm{Er}^{3+}$ Ions
}

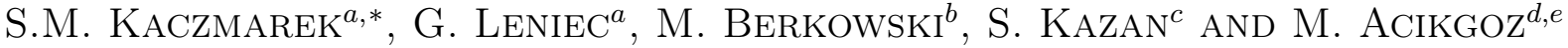 \\ ${ }^{a}$ Institute of Physics, Faculty of Mechanical Engineering and Mechatronics, \\ Westpomeranian University of Technology, 17 Al. Piastow Str., 70-310 Szczecin \\ ${ }^{b}$ Institute of Physics, Polish Academy of Sciences, Aleja Lotnikow 32/46 PL-02668 Warsaw, Poland \\ ${ }^{c}$ Gebze Technical University, Department of Physics, TR-41400 Gebze, Turkey \\ ${ }^{d}$ Bahcesehir University, Faculty of Engineering and Natural Sciences, Beşiktaş, Istanbul, Turkey \\ ${ }^{e}$ Department of Chemistry, Rutgers University, Newark, New Jersey 07102, USA
}

\begin{abstract}
Electron paramagnetic resonance and magnetic susceptibility measurements were performed for two $\mathrm{La}_{3} \mathrm{Ga}_{5.5} \mathrm{Ta}_{0.5} \mathrm{O}_{14}$ single crystals doped with 1.96 at.\% of $\mathrm{Sm}^{3+}$ ions and 3.8 at.\% of $\mathrm{Er}^{3+}$ ions. Two magnetically inequivalent sites were found to be responsible for the electron paramagnetic resonance spectra of $\mathrm{Sm}^{3+}$ ions. Both sites reveal a low (at least $\mathrm{C}_{2}$ ) symmetry with $g_{x} \sim 0.46, g_{y} \sim 0.46$ and $g_{z} \sim 0.52$. The symmetry of isolated erbium ions is higher than $C_{2}$. From electron paramagnetic resonance and magnetic susceptibility experiments it results that the ground state of $\mathrm{Er}^{3+}$ ions is $\Gamma^{6}$ or $\Gamma^{7}$. Below $51 \mathrm{~K}$ electronic transitions within $\Gamma^{6}$ doublets take place. Between 51 and $60 \mathrm{~K}, \Gamma^{6}$ to $\Gamma^{7}$ transitions dominate, while above $60 \mathrm{~K}$ mainly transitions within $\Gamma^{7}$ doublet are active.
\end{abstract}

DOI: 10.12693/APhysPolA.131.366

PACS/topics: 75.10.Dg, 76.30.Kg, 75.30.Gw, 75.40.Cx

\section{Introduction}

The $\mathrm{La}_{3} \mathrm{Ga}_{5.5} \mathrm{Ta}_{0.5} \mathrm{O}_{14}$ (LGT), is a compound of the langasite family [1]. It has been established that LGT forms optically uni-axial crystals belonging to the P321 space group [2]. LGT undergoes no structural phase transitions up to its melting point $\left(1510^{\circ} \mathrm{C}\right)[3,4]$. In the crystal structure of LGT two kinds of atoms [Ga1 and Ta $(0,0,0)]$ reside on the same (octahedral) site in different proportions, giving rise to a certain structural disorder. The local symmetry of the sites is $D_{3}$. Besides, there is one site of $C_{3}$ [(1/3,2/3, z), Ga2 tetrahedron] symmetry and two sites of $C_{2}$ local symmetry [La (x, 0,0$)$, Thomson cube and [Ga3 (x, 0,1/2), trigonal-pyramidal], where $x=0.42492(7)(\mathrm{La}), x=0.7617(1)$ (Ga3) and $z=0.4689(2)[5,6]$.

Rare-earth doped LGT crystals are promising materials for high-power solid state lasers, emitting in the $1 \mu \mathrm{m}$ range [7]. Yb-doped materials are superior to traditional neodymium ones. Co-activation of the Yb-doped crystals with holmium ions leads to appearance of visible luminescence, which is explained by the $\mathrm{Yb}^{3+}-\mathrm{Ho}^{3+}$ stepwise up-conversion mechanism [8-10]. In the present work we deal with magnetic properties of the two other LGT single crystals doped with $\mathrm{Sm}^{3+}[11]$ and $\mathrm{Er}^{3+}$ [12]. In the LGT:Sm${ }^{3+}$ crystal a visible luminescence is possible, taking origin from the metastable ${ }^{4} \mathrm{G}_{5 / 2}$ state to ${ }^{6} \mathrm{H}_{5 / 2}(\sim 550 \mathrm{~nm}),{ }^{6} \mathrm{H}_{7 / 2}(\sim 600 \mathrm{~nm})$ and

*corresponding author; e-mail: skaczmarek@zut.edu.pl
${ }^{6} \mathrm{H}_{9 / 2}(\sim 646 \mathrm{~nm})$ states [11]. It was found that absorption and luminescence transitions exhibit strong anisotropy. Inhomogeneous broadening of the spectral bands was attributed to certain structural disorder of the host, resulting from the substitution of octahedrally coordinated $\mathrm{Ga}^{3+}$ ions by $\mathrm{Ta}^{5+}$ ions [11]. It was concluded that the LGT: $\mathrm{Sm}^{3+}$ system is promising for application as an orange-red emitting phosphor and can show yellow laser emission. In the LGT:Er crysta infrared emission is possible, based on the ${ }^{4} \mathrm{I}_{13 / 2} \rightarrow{ }^{4} \mathrm{I}_{15 / 2}$ transition near $1.6 \mu \mathrm{m}$ and up-conversion of infrared radiation into green luminescence, originated in the ${ }^{2} \mathrm{H}_{11 / 2},{ }^{4} \mathrm{~S}_{3 / 2}$ levels [12]. It was found that inhomogeneous broadening of $\mathrm{Er}^{3+}$ spectral lines improves optical pumping efficiency at $980 \mathrm{~nm}$ by laser diodes, allows for some tuning of laser wavelength and offers generation of ultrashort pulses.

When doping the LGT structure with $\mathrm{Er}^{3+}$ $(1.14 \AA)$ [13] or $\mathrm{Sm}^{3+}(1.23 \AA)$ [11] ions, one can expect that both types of ions enter substitutionally into $\mathrm{La}^{3+}(1.30 \AA)$ site with low $C_{2}$ symmetry. However it is not obvious. EPR is a sensitive tool to study local environment around the probe ion and to estimate spin-Hamiltonian parameters of rare-earth ions in host crystals. There is a necessity to investigate the crystals by applying also MS measurements.

\section{Experimental}

Single crystals of LGT were grown by the Czochralski method [11, 12]. X-ray powder diffraction method has revealed that the crystals crystallize in the trigonal structure, belonging to the $\mathrm{D}_{3}^{2}$ (P321) space group [2] with the lattice cell parameters $a=8.228(2) \AA, c=5.124(2) \AA$. The unit cell comprises one formula unit. 
Two kinds of LGT single crystals, free from inclusions and bubbles, doped with nominal $\mathrm{Sm}^{3+}$ concentration of 3 at.\%, and, $\mathrm{Er}^{3+}$ concentration of 5 at.\% were grown by the Czochralski method along $c$ crystallographic axis. Examination of chemical composition of manufactured crystals by coupled plasma emission spectroscopy has revealed that actual $\mathrm{Sm}^{3+}$ concentration is 1.96 at.\% while $\mathrm{Er}^{3+}$ concentration is 3.8 at. $\%$.

The EPR spectroscopic measurements were carried out using a standard X-band Bruker E500 EPR spectrometer with the field range of $0-1.4 \mathrm{~T}$, the microwave field modulation of $100 \mathrm{kHz}$ in temperature range of $3-300 \mathrm{~K}$.

The static magnetization measurements as a function of temperature and magnetic field were carried out using a Vibrating Sample Magnetometer (PPMS by Quantum Design Corp.). The field and temperature dependences (10-300 K) of magnetization were recorded in zero-field cooled (ZFC) and field cooled (FC) regimes in an applied field of 100 Oe.

\section{Results}

\subsection{EPR results}

$\mathrm{Sm}^{3+}$ ions have a $4 \mathrm{f}^{5}$ electronic configuration. The ground state is ${ }^{6} \mathrm{H}_{5 / 2}$. Spin Hamiltonian of samarium ion in the LGT crystal lattice under an external magnetic field can be written as:

$$
H=\mu_{\mathrm{B}} \boldsymbol{g}_{e} \boldsymbol{B} \boldsymbol{S}+\mu_{\mathrm{B}} \boldsymbol{g}_{n} \boldsymbol{B} \boldsymbol{I}+\boldsymbol{S} \boldsymbol{D}+\boldsymbol{S} \boldsymbol{A} \boldsymbol{I},
$$

where first two terms are Zeeman terms for electron and nucleus, the third and fourth terms are fine structure and hyperfine structure terms, respectively, $\mu_{\mathrm{B}}-$ Bohr magneton, $\boldsymbol{g}_{e}$ and $\boldsymbol{g}_{n}-g$-factors, $\boldsymbol{B}$ - magnetic field, $\boldsymbol{S}$ - electron spin operator, $S=5 / 2$, spin of $\mathrm{Sm}^{3+}$ ion, $\boldsymbol{I}$ - nucleus spin operator, $I=7 / 2$, spin of its nucleus, $\boldsymbol{D}$ - tensor of fine interaction, $\boldsymbol{A}$ - tensor of hyperfine interaction.

$\mathrm{Er}^{3+}$ ions have $4 \mathrm{f}^{11}$ electronic configuration. The ground state, ${ }^{4} \mathrm{I}_{15 / 2}$, is 16 -fold degenerated. It is split into two doublets $\Gamma^{6}, \Gamma^{7}$ and three quartets $\Gamma^{8}$. At a low symmetry these quartets split into doublets, which is a source of strong Zeeman anisotropy. Spin Hamiltonian for an $\mathrm{Er}^{3+}$ ion in the LGT crystal lattice under external magnetic field can be written as:

$$
H=\mu_{\mathrm{B}} \boldsymbol{g} \boldsymbol{B} \boldsymbol{S},
$$

where $H$ is an effective spin Hamiltonian for isolated erbium ions with an effective spin $S_{\text {eff }}=1 / 2$. Remaining parameters have the same meaning as above.

EPR spectra of LGT:Sm ${ }^{3+}$ crystal (Fig. 1a, ac plane) contain a broad spectrum derived from the fine structure, centered at $g_{\text {eff }} \sim 0.5$, being a superposition of at least three bands of six and several bands attributed to hyperfine structure. Calculated value of hyperfine constant, $248 \mathrm{Gs}$ has been assigned to ${ }^{147} \mathrm{Sm}^{3+}$, while hyperfine constant of ${ }^{149} \mathrm{Sm}^{3+}$ is equal to 200 Gs. A ratio of the hyperfine structure parameters of ${ }^{147} \mathrm{Sm}$ and ${ }^{149} \mathrm{Sm}$ isotopes is 1.24 , which is in good agreement with the ratio of the respective magnetic moments. In the figure, temperature dependence of the EPR MS, calculated as double integral of the EPR-measured spectrum, is also presented. Simulation of the dependences using Curie-Weiss law has allowed us to calculate Curie-Weiss temperatures $(0.98 \mathrm{~K}, 3.08 \mathrm{~K},-1.19 \mathrm{~K}$ for $\boldsymbol{a} \boldsymbol{b}, \boldsymbol{a c}$ and $\boldsymbol{b} \boldsymbol{c}$ planes, respectively). The above values indicate on magnetic anisotropy of the investigated crystal.
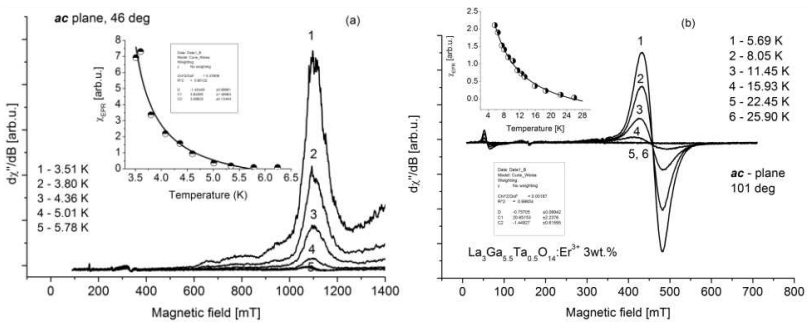

Fig. 1. EPR spectrum in $\boldsymbol{a c}$ plane for several temperatures: (a) LGT:Sm single crystal, $T_{\mathrm{CW}}=3.08 \mathrm{~K}$, (b) LGT:Er single crystal, $T_{\mathrm{CW}}=-2.14 \mathrm{~K}$; The insets show EPR MS (points), fitted using Curie-Weiss law (solid lines).

TABLE I

Normalized values of spin Hamiltonian parameters calculated for LGT:Sm. The $\varphi$ is the angle between the laboratory axes system and crystal axes system.

\begin{tabular}{c|c|c}
\hline \hline$g_{x}^{e}$ & $g_{y}^{e}$ & $g_{z}^{e}$ \\
$0.46(1)$ & $0.46(1)$ & $0.52(1)$ \\
$\varphi=17.2 \mathrm{deg}$. & & \\
\hline$D_{x}[\mathrm{Gs}]$ & $D_{y}[\mathrm{Gs}]$ & $D_{z}[\mathrm{Gs}]$ \\
$20(2)$ & $50(2)$ & $-70(2)$ \\
\hline$g_{x}^{n}$ & $g_{y}^{n}$ & $g_{z}^{n}$ \\
$0.48(1)$ & $0.48(1)$ & $0.52(1)$ \\
\hline$A_{x}[\mathrm{Gs}]$ & $A_{y}[\mathrm{Gs}]$ & $A_{z}[\mathrm{Gs}]$ \\
$260(5)$ & $260(5)$ & $225(5)$
\end{tabular}

The shape of the Eq. 1 allows to analyze magnetic interactions between $\mathrm{Sm}^{3+}$ ions in LGT: $\mathrm{Sm}^{3+}$ crystal using e.g. NMR-EPR program [14]. We have applied the procedure to find spin Hamiltonian parameters (Table I).

From the angular dependences $(\sim 5 \mathrm{~K})$ of LGT:Sm EPR spectra registered in all, $\boldsymbol{a} \boldsymbol{b}, \boldsymbol{a c}$ and $\boldsymbol{b} \boldsymbol{c}$ planes, it results, that at least two kinds of lines are needed to fit experimental measurements. The first one we have attributed to fine structure lines of samarium ions with electron spin $S=5 / 2$, and the second one, to hyperfine structure of samarium ions with nucleus spin $I=7 / 2$. Moreover, we have found two kinds of magnetically different $\mathrm{Sm}^{3+}$ ion positions in LGT lattice. The local symmetry of both sites seems to be $C_{2}$. The spin Hamiltonian parameters are typical to those of $\mathrm{Sm}^{3+}$ ions, reported in other single crystals. E.g., $g_{\|}=0.410$ and $g_{+}=0.644, A_{\|}\left({ }^{147} \mathrm{Sm}\right)=65$ Gs and $A_{+}\left({ }^{147} \mathrm{Sm}\right)=245$ Gs, $A_{\|}\left({ }^{149} \mathrm{Sm}\right)=55$ Gs and $A_{+}\left({ }^{149} \mathrm{Sm}\right)=202$ Gs for $\mathrm{LiYF}_{4}: \mathrm{Sm}^{3+}[15,16]$.

As one can see from Fig. 1b, in the EPR spectra of LGT: $\mathrm{Er}^{3+}$ single crystal there are present some signals 
in a range of low and higher magnetic fields. The signals are observed only below $\sim 30 \mathrm{~K}$ and originate from the Kramers-doublet transitions, $\Gamma^{6}$ or $\Gamma^{7}$, within even-like Er isotopes with an effective spin $S=1 / 2$ and nuclear magnetic moment $I=0$. The EPR signal from the oddlike, 167 isotope, is observed only for isolated and small amounts of erbium dopants. The spectra reveal high magnetic anisotropy of $g$-value, mainly due to dipoledipole interaction. The miscellaneous $g$-values indicate complex interactions between ground and excited states. There may be possible the crossing between split $\Gamma^{6}$ and $\Gamma^{7}$ levels (Fig. 2a) due to strong crystal field or pairing of erbium ions (Fig. 2b), as a result of point defects present in the vicinity of erbium ions. The main EPR signal originating from isolated erbium ions comes from the ground states $\Gamma^{6}$ or $\Gamma^{7}$.
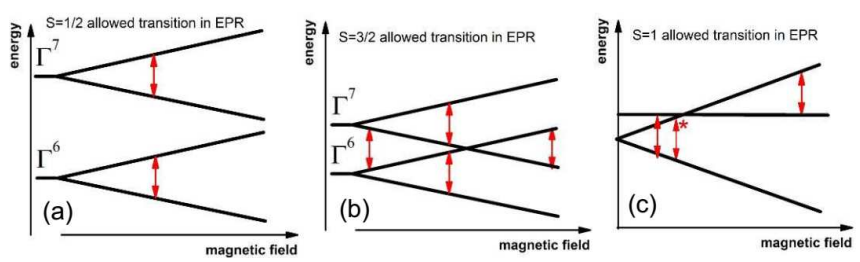

Fig. 2. Simplified energy diagrams of low-lying levels for isolated (a, b) and paired (c) erbium ions [17]. Double-sided arrows represent the possible EPR transitions.

\section{TABLE II}

Spin Hamiltonian parameters of $\mathrm{Er}^{3+}$ ion in LGT crystal. The $g$-parameter can be written as $g_{x}=g_{y}=g_{+}$and $g_{z}=g_{\|}$.

\begin{tabular}{c|c|c|c}
\hline \hline$g_{x}$ & $g_{y}$ & $g_{z}$ & $\bar{g}$ \\
\hline 9.20 & 9.20 & 1.52 & 6.64
\end{tabular}

From angular dependencies $(\sim 5 K)$ of the LGT:Er EPR spectra it results that these spectra consist of several EPR lines (at least four), depending on the selected plane and the angle $\varphi$ (an angle between the direction of magnetic field and the main axes of the crystal). The most intense EPR signal was observed in the $\boldsymbol{a c}$ and $\boldsymbol{b} \boldsymbol{c}$ planes. It modifies its resonance position from $70 \mathrm{mT}$ to $450 \mathrm{mT}$, depending on the angle $\varphi$. The intensity of the signal is much lower in the $\boldsymbol{a} \boldsymbol{b}$ plane. This signal comes from isolated erbium ions at low symmetry, with an effective spin of $S=1 / 2$. Other three EPR signals of low intensity, observed in all planes at positions: $\sim 50 \mathrm{mT}, \sim 190 \mathrm{mT}, \sim 240 \mathrm{mT}$, are not dependent on the magnetic field. The $190 \mathrm{mT}$ signal does not come from erbium ions. It appears usually in doped LGT crystals and may be derived from the disturbance of the crystal lattice caused by a dopant, due to the difference of ionic radii between the dopant and host ion. The other two signals, at 50 and $240 \mathrm{mT}$, may be assigned to pairs of erbium ions, resulting from the crystal lattice dislocations. Similar conclusions were reported by Bodziony et al. [13], however they assumed that signals observed at low fields are assigned to an unknown impurity. They have investigated, however, LGT single crystals with lower concentration of erbium (0.1 at.\%). The angular dependencies were fitted by using Voight lines. The shape of these lines suggests domination of dipolar interactions between erbium ions. The angular dependencies indicate, unexpectedly, that local symmetry of erbium ions in LGT crystal is higher than $C_{2}$. Spin Hamiltonian parameters obtained from the dependencies applying EPRNMR program [14] are presented in Table II.

The average value of $g$-factor was obtained from the following formula: $\bar{g}=\left(g_{x}+g_{y}+g_{z}\right) / 3$. Value $\bar{g}$ indicates that EPR signal originates from $\Gamma^{6}$ ground state. So, the temperature dependencies, registered in the low temperature range describe transitions mainly within the $\Gamma^{6}$ ground state. The $g$-values confirm strong anisotropy of magnetic properties of the LGT:Er crystal.

\subsection{Magnetization measurements}

The dependences of the magnetization in the applied magnetic field for the samples studied in this work have been analyzed via the magnetic hysteresis loops. Hysteresis loops of the $\mathrm{Sm}^{3+}$-doped LGT crystals were measured at 25 and $50 \mathrm{~K}$ in a magnetic field up to $\pm 7 \mathrm{kOe}$. The magnetic hysteresis loops of the samples reveal that no clear saturation magnetization and coercive field are observed for this sample at low temperatures. It is also observed that the magnetization is not sensitive to temperature. The temperature dependences of magnetization recorded in $\mathrm{ZFC}$ and $\mathrm{FC}$ are not overlapping over the entire range of temperatures up to $300 \mathrm{~K}$. It reveals a strong coupling regime by the presence of a strong coupling of the $\mathrm{Sm}^{3+}$ ions to the lattice of LGT crystals.

The hysteresis loops of $\mathrm{Er}^{3+}$-doped LGT crystals have been measured at temperatures $30 \mathrm{~K}$ and $50 \mathrm{~K}$. In general, a remarkable sensitivity to the temperature is observed for the magnetization. As the temperature decreases the magnetization increases for the crystals. The ZFC and FC curves are overlapping over entire range of temperatures up to $300 \mathrm{~K}$. From this behavior we cannot state a strong coupling between $\mathrm{Er}^{3+}$ ions and the lattice of LGT crystals. The small difference between the FC and ZFC indicates that possible pairs of erbium ions may be caused by dislocations of the LGT crystal lattice. From the temperature dependence of the magnetic moment it results that below $50 \mathrm{~K}$ electronic transitions take place within $\Gamma^{6}$ ground state, between 50 and $61 \mathrm{~K}$ (constant value of magnetic moment) electronic transitions between $\Gamma^{6}$ and $\Gamma^{7}$ dominate, while above $61 \mathrm{~K}$ electronic transitions within $\Gamma^{7}$ prevail.

\section{Conclusions}

Two magnetically inequivalent sites were found to be responsible for the EPR spectra of LGT:Sm single crystal. Both sites reveal low symmetry with $g_{x} \sim g_{y} \sim 0.46$ and $g_{z} \sim 0.52$. The spectra are represented mainly by fine and hyperfine structures. Effective hyperfine constant is found to be equal to 248 Gs for ${ }^{147} \mathrm{Sm}^{3+}$ isotope 
and $200 \mathrm{Gs}$ for ${ }^{149} \mathrm{Sm}^{3+}$ one, which means that the ratio of the hyperfine structure parameters of ${ }^{147} \mathrm{Sm}$ and ${ }^{149} \mathrm{Sm}$ isotopes is 1.24. From magnetization measurements it results strong coupling regime related with the presence of a strong coupling of the $\mathrm{Sm}^{3+}$ ions to the lattice of LGT crystals.

The EPR measurements of LGT:Er single crystal show a large anisotropy of isolated centers of erbium ions with weak antiferromagnetic interactions. Based on the spin Hamiltonian parameters we have established symmetry of isolated erbium ions as being higher than $\mathrm{C}_{2}$. Apart from isolated centers of erbium ions we have observed a small amount of pairs of erbium ions, resulting from the LGT crystal lattice dislocation, provided by the difference between the ionic radii of lanthanum and erbium ions. The MS has confirmed the results of the EPR measurements. In the whole temperature range of MS measurements the observed signal comes from isolated erbium ions. For the lowest temperatures up to $T=50 \mathrm{~K}$ the signal seems to originate from transitions within $\Gamma^{6}$ ground state, in the range of $50<T<61 \mathrm{~K}$, from transitions between $\Gamma^{6}$ and $\Gamma^{7}$ level, and above $61 \mathrm{~K}$ from the transitions within the $\Gamma^{7}$ level.

\section{References}

[1] E.L. Belokoneva, A.V. Butashin, M.A. Simonov, B.V. Mill, N.V. Belov, Dokl. Akad. Nauk SSSR 25, 954 (1980).

[2] H. Takeda, K. Sugiyama, K. Inava, K. Shimamura, T. Fukuda, Jpn. J. Appl. Phys. 36, L919 (1997).

[3] A. Kaminskii, I. Silvestrova, S. Sarkisov, G. Denisenko, Phys. Stat. Sol. A 80, 607 (1983).
[4] A. Kaminskii, B. Mill, G. Khodzhabagyan, A. Konstantinova, A. Okorochkov, I. Silvestrova, Phys. Stat. Sol. A 80, 387 (1983).

[5] J. Bohm, R.B. Heimann, M. Hengst, R. Roewer, J. Schindler, J. Cryst. Growth 204, 128 (1999).

[6] H. Fuks, S.M. Kaczmarek, M. Berkowski, Phys. Stat. Sol. A 203, 3640 (2006).

[8] L.F. Johnson, H.J. Guggenheim, Appl. Phys. Lett. 19, 44 (1971)

[9] Th. Rothacher, W. Luthy, H.P. Weber, Optics Comm. 155, 68 (1998).

[10] R. Lisiecki, W. Ryba-Romanowski, L. Macalik, J. Komar, M. Berkowski, Appl. Phys. B 116, 183 (2014).

[11] J. Komar, R. Lisiecki, W. Ryba-Romanowski, M. Berkowski, J. Alloys. Comp. 610, 50 (2014).

[12] R. Lisiecki, W. Ryba-Romanowski, J. Komar, M. Berkowski, J. Alloys. Comp 610, 451 (2014).

[13] T. Bodziony, J. Typek, M. Orlowski, J. Majszczyk, M. Wabia, M. Berkowski, W. Ryba-Romanowski, N. Guskos, V. Likodimos, E.A. Anagnostakis, Acta Phys. Pol. A 103, 315 (2003).

[14] M.J. Mombourquette, J.A. Weil, D.G. McGavi, EPRNMR User's manual, Department of Chemistry, University of Saskatchewan, Saskatoon 1999.

[15] M.L. Falin, K.I. Gerasimov, N.M. Khaidukov, Appl. Magn. Reson. 40, 31 (2011).

[16] D. Hui-Ning, Z. Wen-Chen, W. Shao-Yi, T. Sheng, Spectrochim. Acta A 60, 489 (2004).

[17] A. Abragam, B. Bleaney, Electron Paramagnetic Resonance of Transition Ions, Oxford University Press 1970. 